Real - ti me at omi c- resol uti on i magi ng of crystal growth process in water by phase nodul at i on at om c force microscopy at one frame per second

\begin{tabular}{|l|l|}
\hline 著者 & M yat a Kazuki, Asakawa H toshi, Fukuna Takeshi \\
\hline 著者別表示 & 宮田 一輝, 淺川 雅, 福間 岡士 \\
\hline $\begin{array}{l}\text { j our nal or } \\
\text { publ i cat i on t i t l e }\end{array}$ & Appl i ed Physi cs Letter s \\
\hline vol une & 103 \\
\hline number & 20 \\
\hline page range & 203104 \\
\hline year & 2013 11- 11 \\
\hline URL & ht t p: //doi .org/10. 24517/00008495 \\
\hline
\end{tabular}




\title{
Real-time atomic-resolution imaging of crystal growth process in water by phase modulation atomic force microscopy at one frame per second
}

\author{
Kazuki Miyata, ${ }^{1}$ Hitoshi Asakawa, ${ }^{2}$ and Takeshi Fukuma ${ }^{1,2,3, a)}$ \\ ${ }^{1}$ Division of Electrical Engineering and Computer Science, Kanazawa University, Kakuma-machi, \\ Kanazawa 920-1192, Japan \\ ${ }^{2}$ Bio-AFM Frontier Research Center, Kanazawa University, Kakuma-machi, Kanazawa 920-1192, Japan \\ ${ }^{3}$ ACT-C, Japan Science and Technology Agency, Honcho 4-1-9, Kawaguchi 332-0012, Japan
}

(Received 3 September 2013; accepted 29 October 2013; published online 11 November 2013)

\begin{abstract}
Recent advancement in dynamic-mode atomic force microscopy (AFM) has enabled its operation in liquid with atomic-scale resolution. However, its imaging speed has often been too slow to visualize atomic-scale dynamic processes. Here, we propose a method for making a significant improvement in the operation speed of dynamic-mode AFM. In this method, we use a wideband and low-latency phase detector with an improved algorithm for the signal complexification. We demonstrate atomic-scale imaging of a calcite crystal growth process in water at one frame per second. The significant improvement in the imaging speed should enable various studies on unexplored atomic-scale interfacial processes. (C) 2013 AIP Publishing LLC.

[http://dx.doi.org/10.1063/1.4830048]
\end{abstract}

Atomic force microscopy (AFM) ${ }^{1}$ is capable of imaging atomic-scale structures even in liquid. Thus, its applications to the studies on dynamic phenomena at solid/liquid interfaces have intensively been explored. So far, high-speed amplitude modulation AFM (AM-AFM) has been developed. ${ }^{2,3}$ It has enabled direct imaging of dynamic biological processes at nanoscale resolution. ${ }^{4,5}$ However, its spatial resolution has been limited to a few nanometers. In contrast, contact-mode AFM has traditionally been used for atomicscale imaging in liquid. ${ }^{6}$ It has widely been used for the studies on crystal growth processes of various minerals. ${ }^{7,8}$ However, the atomic-scale contrast obtained by contactmode AFM are often averaged over nanoscale area. ${ }^{9}$ In addition, the lateral friction force caused by the tip scan often prevents non-destructive imaging of soft materials.

Non-destructive imaging with true atomic-resolution has routinely been performed by dynamic-mode AFM with a relatively stiff cantilever (spring constant $k>10 \mathrm{~N} / \mathrm{m}$ ). ${ }^{10,11}$ While the method has traditionally been used in vacuum, its operation in liquid with true atomic resolution has recently become possible. ${ }^{12}$ The high spring constant of the cantilever enables to avoid the tip adhesion event referred to as jumpto-contact. In addition, it provides lower thermal vibration amplitude of the cantilever. Thus, we can precisely control the vertical tip position in the atomic-scale non-contact regime. Owing to this capability, the method allows visualizing not only surface structures but also three-dimensional (3D) distribution of mobile water (i.e., hydration structures). ${ }^{13,14}$ However, the imaging speed of the method has been limited to less than one frame per min.

To enhance the speed of the present dynamic-mode AFM without deteriorating the force and spatial resolutions, we should enhance both the minimum detectable force $\left(F_{\min }\right)$ and the tip-sample distance feedback bandwidth $\left(B_{F B}\right)$. Recently, we have developed a method to stably control the oscillation of a small cantilever with a resonance

${ }^{\text {a)} E l e c t r o n i c ~ m a i l: ~ f u k u m a @ s t a f f . k a n a z a w a-u . a c . j p ~}$ frequency $\left(f_{0}\right)$ of $\sim 3 \mathrm{MHz}$ in liquid. ${ }^{15}$ With the small cantilever, we can obtain $\sim 7.3$ times smaller $F_{\min }$ than that obtained with a conventional cantilever $\left(f_{0}=130 \mathrm{kHz}\right.$ in liquid). ${ }^{15}$ As $F_{\text {min }}$ is proportional to $\sqrt{B_{\mathrm{FB}}}$, the small cantilever should allow us to achieve 50 times wider $B_{F B}$ with the same $F_{\text {min }}$ as that obtained with a conventional cantilever. The typical $B_{F B}$ used for the atomic-resolution imaging by conventional dynamic-mode AFM is $10-100 \mathrm{~Hz}$. Thus, the 50 times wider $B_{\mathrm{FB}}$ corresponds to $0.5-5 \mathrm{kHz}$.

To enhance $B_{F B}$, it is necessary to improve the bandwidth or the resonance frequency of all the components constituting the tip-sample distance regulation feedback loop. Among them, a phase detector (PD) is one of the key components in dynamic-mode AFM with true atomic resolution such as phase modulation AFM (PM-AFM) ${ }^{16}$ and frequency modulation AFM (FM-AFM). ${ }^{17}$ A lock-in amplifier with a multiplication-based phase comparator is most commonly used as a PD in AFM. In the design, however, a low-pass filter (LPF) with a relatively large latency is used for eliminating the higher harmonics generated by the multiplication of the input and reference signals. Thus, its application to highspeed AFM is difficult.

To overcome this problem, Stark and Guckenberger developed a high-speed PD using a sample and hold circuit (SH-PD). ${ }^{18}$ In the design, an instantaneous phase value is sampled once per oscillation cycle. Uchihashi et al. used an SH-PD with high-speed AM-AFM to perform high-speed phase imaging. ${ }^{19}$ Sugawara et al. developed high-speed PM-AFM using an SH-PD. ${ }^{20,21}$ They also combined it with a multifrequency technique and demonstrated high-speed elasticity mapping. ${ }^{22}$

While these previous works demonstrated the fast time response of an SH-PD, its applicability to atomic-scale imaging has not been demonstrated. The phase output of an SH-PD shows a discontinuous jump once per oscillation cycle and hence contains large spurious components at frequencies corresponding to the multiple of the cantilever oscillation frequency. These spurious components can 


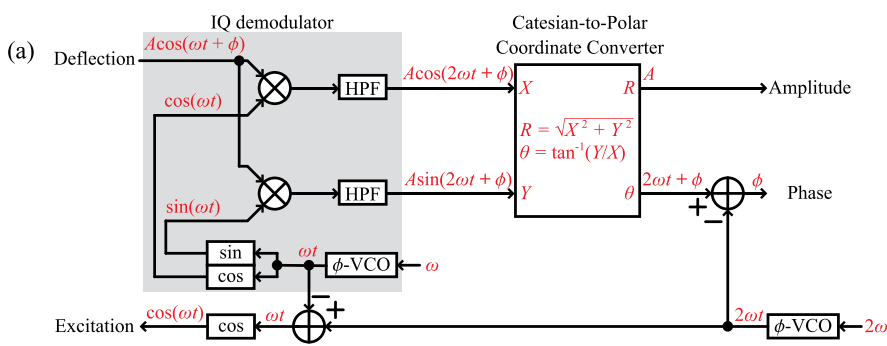

prevent the precise tip positioning that is essential for true atomic-resolution imaging.

We have recently proposed another design of a highspeed PD using a subtraction-based phase comparator (SP-PD). ${ }^{23}$ In the design, the input signal is converted to in- and quadratic-phase (IQ) signals by an IQ demodulator using a Hilbert transformer. The argument of the complex signal is compared with the phase of the cantilever excitation signal with a subtraction-based phase comparator. The SP-PD does not require any LPFs for the IQ demodulation and hence has a wide bandwidth. In addition, the phase output of the SP-PD shows no discontinuous jumps. Thus, it is applicable to atomic-resolution imaging. ${ }^{23}$ However, its application to high-speed imaging has been hindered by the large latency of the Hilbert transformer.

In this study, we propose a method for making a remarkable improvement in the operation speed of dynamic-mode AFM using a wideband and low latency PD. We present an improved algorithm that enables IQ demodulation with an extremely low latency. With the proposed method, we demonstrate high-speed and atomic-resolution imaging of calcite crystal growth process in water at one frame per second.

Figure 1(a) shows a block diagram of the PD used for the high-speed AFM imaging. In the PD, the cantilever deflection signal $A \cos (\omega t+\phi)$ is fed into the IQ demodulator. $A, \omega$ and $\phi$ denote amplitude, frequency, and phase of the cantilever oscillation, respectively. In the IQ demodulator, the input signal is multiplied by the reference signals $[\cos (\omega t)$ and $\sin (\omega t)]$ and converted to dc and $2 \omega$ components. While the dc components are rejected by high-pass filters (HPFs), the $2 \omega$ components $[A \cos (2 \omega t+\phi)$ and $A \sin (2 \omega t+\phi)]$ are passed to a Cartesian-to-Polar coordinate converter. In the circuit, amplitude $(A)$ and phase $(2 \omega t+\phi)$ of the complex signal are calculated.

In the PD, phase signals, $\omega t$ and $2 \omega t$, are generated by phase-output voltage controlled oscillators ( $\phi$-VCOs). The $\omega t$ signal is fed into sine and cosine converters to produce the two reference signals. The $2 \omega t$ signal is subtracted from the $(2 \omega t+\phi)$ signal to produce the final output of the PD $(\phi)$. The difference between the $2 \omega t$ and $\omega t$ signals is calculated and fed into a cosine converter to produce the cantilever excitation signal $[\cos (\omega t)]$.

In this study, we implemented the proposed circuit in a field programmable gate array (FPGA) chip (Vertex-5, Xilinx). The clock frequency of the FPGA was set at $100 \mathrm{MHz}$. The sampling rates of the analog-to-digital and digital-to-analog converters were set at 100 MSPS.

In the proposed algorithm, the significant improvement is achieved by the HPFs. For example, assume that we use a small cantilever with a $f_{0}$ of $3 \mathrm{MHz} .{ }^{15}$ In this case, the $3 \mathrm{MHz}$ input signal is converted to the dc and $6 \mathrm{MHz}$ components. (b)

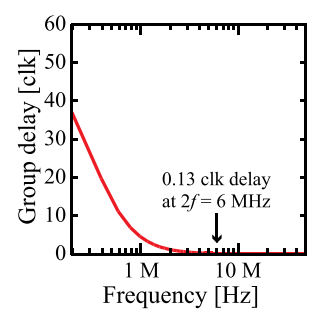

FIG. 1. (a) Schematic diagram of the proposed PD. (b) Group delay of the $\mathrm{HPF}$ for a $3 \mathrm{MHz}$ input signal.

While the dc components are rejected by the HPFs, $6 \mathrm{MHz}$ components are passed through the HPFs. At the pass band frequency, the HPFs have only 0.13 clk group delay as shown in Fig. 1(b). Therefore, this algorithm enables IQ demodulation with almost no delay, which marks remarkable improvement from the previously reported PD. ${ }^{23}$

Figure 2 shows the performance of the proposed PD. We measured modulation and demodulated signals with the input frequency $(f)$ of $3 \mathrm{MHz}$ and the modulation frequency $\left(f_{m}\right)$ of $100 \mathrm{kHz}$ (Fig. 2(a)). The waveform of the demodulated signal shows a clean sine wave profile with a small phase delay. The result shows that the harmonic distortions caused by the demodulation are not significant.

We also measured frequency response of the PD with $f=3 \mathrm{MHz}$ (Fig. 2(b)). The amplitude curve shows flat response up to the input frequency (i.e., $3 \mathrm{MHz}$ ). The result shows that the bandwidth of the PD is limited only by the input frequency. To evaluate the latency, we fitted the phase curve to the equation $\phi=\omega \tau$, where $\tau$ denotes the time delay caused by the PD. The curve is well-fitted to the equation with $\tau=0.97 \mu$ s. This value agrees with the phase difference between the modulation and demodulated signals as shown in Fig. 2(a). The result shows that the phase delay is mostly caused by the fixed latency of the AD/DA conversion and the digital signal processing. The latency of $1 \mu$ s corresponds to the phase delay of $1.8^{\circ}$ at $5 \mathrm{kHz}$. Thus, it is fast enough to achieve $B_{\mathrm{FB}}$ higher than $5 \mathrm{kHz}$.
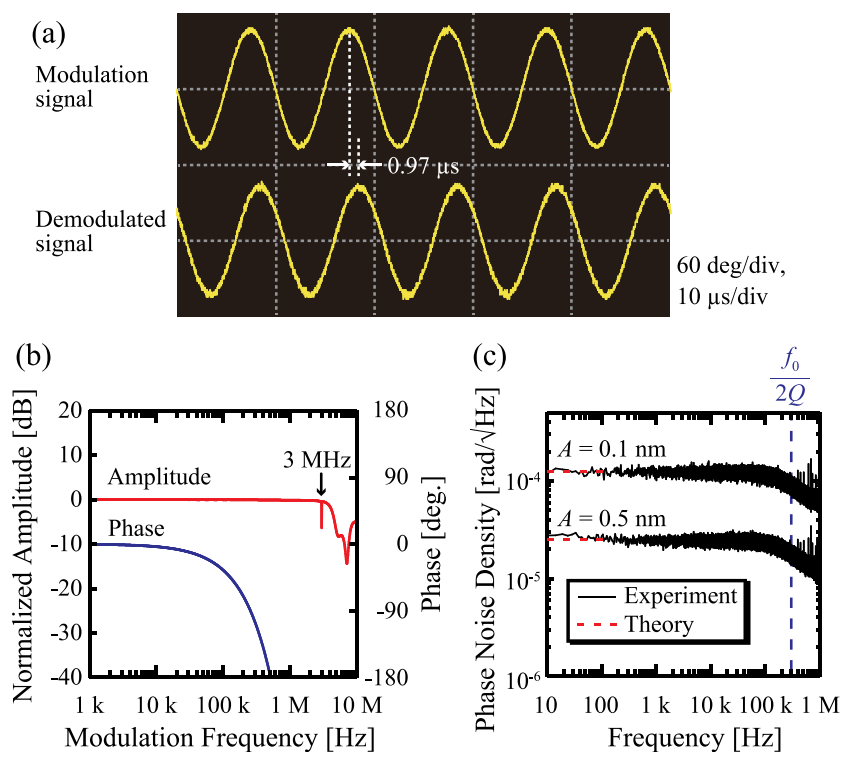

FIG. 2. Performance of the proposed PD. (a) Waveforms of the modulation and demodulated signals $\left(f=3 \mathrm{MHz}, f_{m}=100 \mathrm{kHz}\right)$. (b) Frequency response $(f=3 \mathrm{MHz})$. (c) Phase noise density spectra measured with a small cantilever (USC, Nanoworld) in water ( $\left.k=55.9 \mathrm{~N} / \mathrm{m}, f_{0}=3.19 \mathrm{MHz}, Q=5.4\right)$. 
To evaluate the noise performance, we combined the proposed PD with our custom-built $\mathrm{AFM}^{24-26}$ and performed the following experiment. We oscillated a small cantilever by the photothermal excitation method in water far away from the sample surface. The cantilever deflection signal was fed into the PD. The spectral density distribution of the output phase signal from the PD was measured with amplitude of $A=0.1$ and $0.5 \mathrm{~nm}$ (Fig. 2(c)). These two amplitude values correspond to the minimum and maximum values typically used for atomic-resolution imaging in liquid.

In Fig. 2(c), experimentally measured spectra (solid lines) in the frequency range below $100 \mathrm{kHz}$ show good agreement with the theoretically expected values $n_{\phi}$ (dotted lines) determined by the cantilever thermal vibration. $n_{\phi}$ is given by ${ }^{16}$

$$
n_{\phi}=\sqrt{\frac{4 k_{B} T Q}{\pi f_{0} k A^{2}}},
$$

where $k_{B}$ and $T$ denote Boltzmann constant and absolute temperature, respectively. This result shows that the phase noise from the PD is negligible compared with that caused by the cantilever thermal vibration. Namely, we are able to achieve the thermal-noise-limited performance in PM-AFM using the proposed PD. The decrease of the phase noise above $100 \mathrm{kHz}$ is caused by the decrease of the spectral density of the cantilever thermal vibration above $f_{0} / 2 Q$ $(=300 \mathrm{kHz})$ as indicated in Fig. 2(c).

Figure 3 shows PM-AFM images of a cleaved calcite surface obtained in water using the proposed PD. These images are selected from the 44 successive images taken at $1 \mathrm{~s} /$ frame (see also supplementary video ${ }^{27}$ ). In the images, the lateral motion of the step edge caused by the crystal growth is clearly visualized with atomic resolution. The distance between the adjacent atomic-scale corrugations is $\sim 0.5 \mathrm{~nm}$ while the tip velocity is $2 \mu \mathrm{m} / \mathrm{s}$. Thus, the frequency of the corrugations to be detected $\left(f_{\mathrm{cr}}\right)$ is $\sim 4 \mathrm{kHz}$. This is close to the maximum $B_{\mathrm{FB}}(5 \mathrm{kHz})$ that we can achieve without deteriorating $F_{\min }$ beyond the value obtained by the conventional cantilever.

The speed of the step growth calculated from the images is $0.8 \mathrm{~nm} / \mathrm{s}$. As we used an open liquid cell for this experiment, we cannot precisely determine the solution concentration. According to the previous study, ${ }^{28}$ the step growth rate of calcite is $0.6-0.7 \mathrm{~nm} / \mathrm{s}$ at $150 \mu \mathrm{M}, 1.0-2.7 \mathrm{~nm} / \mathrm{s}$ at $200 \mu \mathrm{M}$, and $3.0-4.6 \mathrm{~nm} / \mathrm{s}$ at $250 \mu \mathrm{M}$. Thus, the solution concentration was probably in the range of $150-200 \mu \mathrm{M}$.

With a typical imaging speed of the conventional PM-AFM $(\sim 1 \mathrm{~min} /$ frame $)$, the step should move by $\sim 8 \mathrm{~nm}$ during one frame scan due to the step growth at $0.8 \mathrm{~nm} / \mathrm{s}$. This should results in a large distortion of the image, making it difficult to understand the true topographic changes. In contrast, with the improved imaging speed ( $1 \mathrm{~s} /$ frame), the step moves only by $0.8 \mathrm{~nm}$ during one frame scan. This is sufficiently small for understanding the true dynamic processes.

In this study, we have proposed a method for making a remarkable improvement in the operation speed of dynamicmode AFM using a wideband and low-latency PD. The proposed PD has a bandwidth of higher than $3 \mathrm{MHz}$ and the
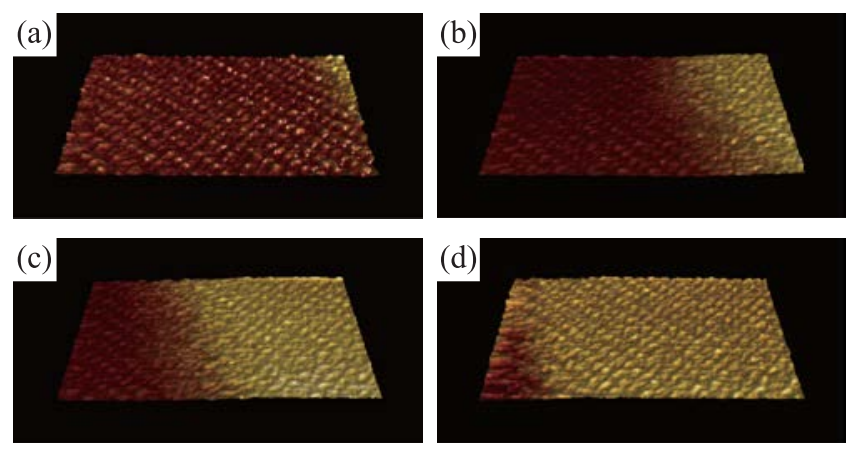

FIG. 3. PM-AFM images of the cleaved calcite surface obtained in water. The images are selected from the 44 successive images obtained at $1 \mathrm{~s} /$ frame (see also supplementary video ${ }^{27}$ ). (a) 0 s. (b) 5 s. (c) 10 s. (d) 15 s. Scan size: $10 \times 5 \mathrm{~nm}^{2}$. Scan rate: $100 \mathrm{~Hz}$. Pixel size: $200 \times 100 \mathrm{pix}^{2}$. The images were obtained with distance feedback regulation in the constant phase shift mode. Thus, they represent the surface height variation. The images show that the calcite step grows from the right to the left.

latency of less than $1 \mu \mathrm{s}$. With the PD, we have demonstrated the real-time imaging of the calcite crystal growth process with atomic resolution. The proposed method should enable in-situ imaging of various interfacial phenomena such as crystal growth, dissolution, and corrosion processes with atomic-scale resolution. In addition, the high-speed IQ demodulator proposed in this study is applicable not only to the phase detection but also to the amplitude and frequency detections. Therefore, it should also enable high-speed operation of AM-AFM and FM-AFM.

This work was supported by ACT-C, Japan Science and Technology Agency and JSPS KAKENHI Grant No. 25706023.

${ }^{1}$ G. Binnig, C. F. Quate, and Ch. Gerber, Phys. Rev. Lett. 56, 930 (1986). ${ }^{2}$ M. B. Viani, T. E. Schäffer, G. T. Paloczi, L. I. Pietrasanta, B. L. Smith, J. B. Thompson, M. Richter, M. Rief, H. E. Gaub, K. W. Plaxco, A. N. Cleland, H. G. Hansma, and P. K. Hansma, Rev. Sci. Instrum. 70, 4300 (1999).

${ }^{3}$ T. Ando, N. Kodera, E. Takai, D. Maruyama, K. Saito, and A. Toda, Proc. Natl. Acad. Sci. U.S.A. 98, 12468 (2001).

${ }^{4}$ N. Kodera, D. Yamamoto, R. Ishikawa, and T. Ando, Nature 468, 72 (2010).

${ }^{5}$ T. Uchihashi, R. Iino, T. Ando, and H. Noji, Science 333, 755 (2011).

${ }^{6}$ F. Ohnesorge and G. Binnig, Science 260, 1451 (1993).

${ }^{7}$ P. E. Hillner, S. Manne, and P. K. Hansma, Faraday Discuss. 95, 191 (1993).

${ }^{8}$ K. Miyata, S. Usho, S. Yamada, S. Furuya, K. Yoshida, H. Asakawa, and T. Fukuma, Rev. Sci. Instrum. 84, 043705 (2013).

${ }^{9}$ J. B. Pethica and W. C. Oliver, Phys. Scr., T 19, 61 (1987).

${ }^{10}$ F. J. Giessibl, Science 267, 68 (1995).

${ }^{11}$ Noncontact Atomic Force Microscopy (Nanoscience and Technology), edited by S. Morita, R. Wiesendanger, and E. Meyer (Springer-Verlag, 2002).

${ }^{12}$ T. Fukuma, K. Kobayashi, K. Matsushige, and H. Yamada, Appl. Phys. Lett. 87, 034101 (2005).

${ }^{13}$ T. Fukuma, Y. Ueda, S. Yoshioka, and H. Asakawa, Phys. Rev. Lett. 104, 016101 (2010).

${ }^{14}$ E. T. Herruzo, H. Asakawa, T. Fukuma, and R. Garcia, Nanoscale 5, 2678 (2013).

${ }^{15}$ T. Fukuma, K. Onishi, N. Kobayashi, A. Matsuki, and H. Asakawa, Nanotechnology 23, 135706 (2012).

${ }^{16}$ T. Fukuma, J. I. Kilpatrick, and S. P. Jarvis, Rev. Sci. Instrum. 77, 123703 (2006).

${ }^{17}$ T. R. Albrecht, P. Grütter, D. Horne, and D. Ruger, J. Appl. Phys. 69, 668 (1991).

${ }^{18}$ M. Stark and R. Guckenberger, Rev. Sci. Instrum. 70, 3614 (1999). 
${ }^{19}$ T. Uchihashi, T. Ando, and H. Yamashita, Appl. Phys. Lett. 89, 213112 (2006).

${ }^{20}$ Y. J. Li, N. Kobayashi, Y. Naitoh, M. Kageshima, and Y. Sugawara, Appl. Phys. Lett. 92, 121903 (2008).

${ }^{21}$ Y. J. Li, N. Kobayashi, H. Nomura, Y. Naitoh, M. Kageshima, and Y. Sugawara, Jpn. J. Appl. Phys., Part 1 47, 6121 (2008)

${ }^{22}$ Y. J. Li, K. Takahashi, N. Kobayashi, Y. Naitoh, M. Kageshima, and Y. Sugawara, Ultramicroscopy 110, 582 (2010).

${ }^{23}$ Y. Mitani, M. Kubo, K. Muramoto, and T. Fukuma, Rev. Sci. Instrum. 80, 083705 (2009).
${ }^{24}$ T. Fukuma, M. Kimura, K. Kobayashi, K. Matsushige, and H. Yamada, Rev. Sci. Instrum. 76, 053704 (2005).

${ }^{25}$ T. Fukuma and S. P. Jarvis, Rev. Sci. Instrum. 77, 043701 (2006).

${ }^{26}$ T. Fukuma, Rev. Sci. Instrum. 80, 023707 (2009).

${ }^{27}$ See supplementary material at http://dx.doi.org/10.1063/1.4830048 for the video consisting of 44 successive PM-AFM images showing the calcite crystal growth process.

${ }^{28}$ A. J. Gratz, P. E. Hillner, and P. K. Hansma, Geochim. Cosmochim. Acta 57, 491 (1992). 